\title{
A polynomial-time approximation scheme for Euclidean Steiner forest*
}

\author{
Glencora Borradaile \\ School of EECS \\ Oregon State University \\ glencora@eecs.orst.edu
}

\author{
Philip N. Klein ${ }^{\dagger}$ \\ Computer Science \\ Brown University \\ klein@brown.edu
}

\author{
Claire Mathieu \\ CNRS \\ Ecole Normale Supérieure \\ cmathieu@di.ens.fr
}

\begin{abstract}
We give a randomized $O(n$ polylog $n)$-time approximation scheme for the Steiner forest problem in the Euclidean plane. For every fixed $\epsilon>0$ and given $n$ terminals in the plane with connection requests between some pairs of terminals, our scheme finds a $(1+\epsilon)$-approximation to the minimum-length forest that connects every requested pair of terminals.
\end{abstract}

\section{Introduction}

\subsection{Result and background}

In the Steiner forest problem, we are given a set of $n$ pairs of terminals $\left\{\left(t_{i}, t_{i}^{\prime}\right)\right\}_{i=1}^{n}$. The goal is to find a minimumcost forest $F$ such that every pair of terminals is connected by a path in $F$. We consider the problem where the terminals are points in the Euclidean plane. The solution is a set of line segments of the plane; non-terminal points with more than two line segments adjacent to them in the solution are called Steiner points. The cost of $F$ is the sum of the lengths in $\ell_{2}$ of the line segments comprising it. Our main result is:

Theorem 1.1. There is a randomized $O(n$ polylog $n)$-time approximation scheme for the Steiner forest problem in the Euclidean plane.

An approximation scheme is guaranteed, for a fixed $\epsilon$, to find a solution whose total length is an most $1+\epsilon$ times the length of a minimum solution.

Independently, Mitchell [16] and Arora [2] developed a method for designing polynomial-time approximation schemes (PTASes) for problems such as such as Traveling Salesman and Steiner tree in the Euclidean plane. The running time for Arora's technique was improved upon by Rao and Smith for the Steiner tree and TSP problems [17] and others have extended these techniques to give PTASes for other problems, e.g., k-medians [4, 15]. Our work builds on the these techniques, using the framework as described by Arora.

The Steiner forest problem, a generalization of the Steiner tree problem, is NP-hard [13] and max-SNP complete [8, 18] in general graphs and high-dimensional Euclidean space [19]. Therefore, no PTAS exists for these problems. The 2-approximation algorithm due to Agrawal, Klein and Ravi [1] can be adapted to Euclidean problems by restricting the Steiner points to lie on a sufficiently fine grid and converting the problem into a graph problem.

We have formulated the connectivity requirements in terms of pairs of terminals. One can equivalently formulate these in terms of sets of terminals: the goal is then to find a forest in which each set of terminals are connected. Arora states [3] that his approach yields an approximation scheme whose running time is exponential in the number of sets

\footnotetext{
${ }^{*}$ This version is more recent than that appearing in the FOCS proceedings. The partition step has been corrected and the overall presentation has been clarified and formalized. This material is based upon work supported by the National Science Foundation under Grant Nos. CCF-0635089, CCF-0964037, and CCF-0963921.

† Work done while visiting MIT's CSAIL.
} 
of terminals, and this is the only previous work to take advantage of the Euclidean plane to get a better approximation ratio than that of Agrawal et al. [1].

\subsection{Recursive dissection}

In Arora's paradigm, the feasible space is recursively decomposed by dissection squares using a randomized variant of the quadtree (Figure 1). The dissection is a 4-ary tree whose root is a square box enclosing the input terminals, whose width $L$ is twice the width of the smallest square box enclosing the terminals, and whose lower left-hand corner of the root box is translated from the lower left-hand corner of the bounding box by $(-a,-b)$, where $a$ and $b$ are chosen uniformly at random from the range $[0, L / 2)$. Each node in the tree corresponds to a dissection square. Each square is dissected into four parts of equal area by one vertical and one horizontal dissection line each spanning the breadth of the root box. This process continues until each square contains at most one terminal (or multiple terminals having the same coordinates).

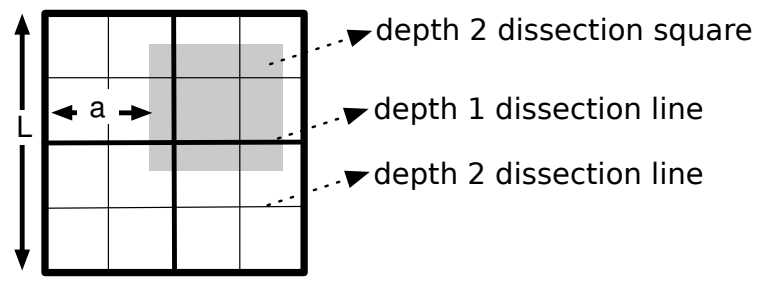

Figure 1: The shifted quad-tree dissection. The shaded box is the bounding box of the terminals.

Feasible solutions are restricted to using a small number of portals, designated points on each dissection line. A Structure Theorem states that there is a near-optimal solution that obeys these restrictions. The final solution is found by a dynamic program guided by the recursive decomposition.

In the problems considered by Arora, the solutions are connected. However, the solution to a Steiner forest problem is in general disconnected, since only paired terminals are required to be connected. It is not known a priori how the connected components partition the terminal pairs. For that reason, maintaining feasibility in the dynamic program requires a table that is is exponential in the number of terminal pairs. In fact, Arora states [3] that his approach yields an approximation scheme whose running time is exponential in the number of sets of terminals.

Nevertheless, here we use Arora's approach to get an approximation scheme whose running time is polynomial in the number of sets of terminals. The main technical challenge is in maintaining feasibility in a small dynamic programming table.

\subsection{Small dynamic programming table}

We will use Arora's approach of a random recursive dissection. Arora shows (ie. for Steiner tree) that the optimal solution can be perturbed (while increasing the length only slightly) so that, for each box of the recursive dissection, the solution within the box interacts weakly and in a controlled way with the solution outside the box. In particular, the perturbed solution crosses the boundary of the box only a constant number of times, and only at an $O(1)$-sized subset of $O(\log n)$ selected points, called portals. The optimal solution that has this property can be found using dynamic programming.

Unfortunately, for Steiner forest those restrictions are not sufficient: maintaining feasibility constraints cannot be done with a polynomially-sized dynamic program. To see why, suppose the solution uses only 2 portals between adjacent dissection squares $R_{E}$ and $R_{W}$. In order to combine the solutions in $R_{W}$ and $R_{E}$ in the dynamic program into a feasible solution in $R_{W} \cup R_{E}$, we need to know, for each pair $\left(t, t^{\prime}\right)$ of terminals with $t \in R_{W}$ and $t^{\prime} \in R_{E}$, which portal connects $t$ and $t^{\prime}$ (Figure 2(a)). This requires $2^{n}$ configurations in the dynamic programming table.

To circumvent the problem in this example, the idea is to decompose $R_{W}$ and $R_{E}$ into a constant number of smaller dissection squares called cells. All terminals in a common cell that go to the boundary use a common portal. Thus, 
(a)
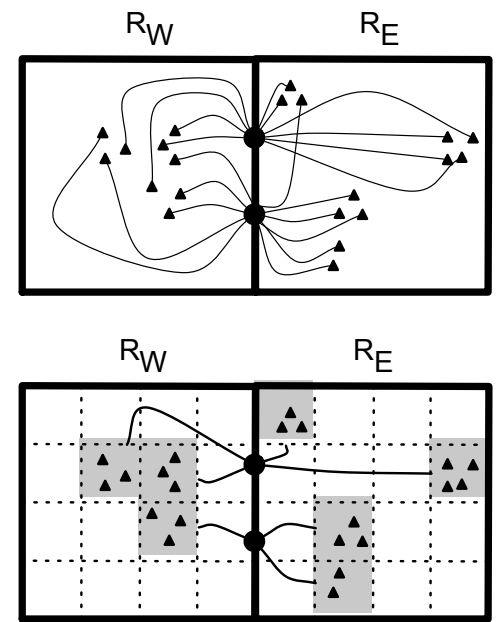

Figure 2: Maintaining feasibility is not trivially polynomial-sized.

instead of keeping track of each terminal's choice of portal individually, the dynamic program can simply memoize each cell's choice of portal. The dynamic program also uses a specification of how portals must be connected outside the dissection squares. This information is sufficient to check feasibility when combining solutions of the subproblems for $R_{W}$ and for $R_{E}$. To show near-optimality, we show that a constant number of cells per square is sufficient for finding a nearly-optimal solution.

Basic notation and definitions For two dissection squares $A$ and $B$, if $A$ encloses $B$, we say that $B$ is a descendent of $A$ and $A$ is an ancestor of $B$. If no other dissection square is enclosed by $A$ and encloses $B$, we say that $A$ is the parent of $B$ and $B$ is the child of $A$. We will extend these definitions to describe relationships between cells. The depth of a square $S$ is given by its depth in the dissection tree ( 0 for the root). The depth of a dissection line is the minimum depth of squares it separates. Note that a square at depth $i$ is bounded by two perpendicular depth- $i$ lines and two lines of depth less than $i$.

For a line segment $s$ (open or closed), we use length $(s)$ to denote the $\ell_{2}$ distance between $s$ 's endpoints. For a set of line segments $S=\left\{s_{1}, s_{2}, \ldots\right\}$, length $(S)=\sum_{i}$ length $\left(s_{i}\right)$. For a subset $X$ of the Euclidean plane, a component of $X$ is a maximal subset $Y$ of $X$ such that every pair of points in $Y$ are path-wise connected in $X$. We use $|X|$ to denote the number of components of $X$. The diameter of a connected subset $C$ of the Euclidean plane, $\operatorname{diam}(C)$, is the maximum $\ell_{2}$ distance between any pair of points in $C$. We use OPT to denote both the line segments forming an optimal solution and the length of those line segments.

\section{The algorithm}

The algorithm starts by finding a rough partition of the terminals which is a coarsening of the connectivity requirements (subsection 2.1. We solve each part of this partition independently. We next discretize the problem by moving the terminals to integer coordinates of a sufficiently fine grid (subsection 2.2). We will also require that the Steiner points be integer coordinates. We next perform a recursive dissection (subsection 2.3) and assign points on the dissection lines as portals (subsection 2.4) as introduced in Section 1.2. We then break each dissection square into a small number of cells. We find the best feasible solution $F$ to the discretized problem that only crosses between dissection squares at portals and such that for each cell $C$ of dissection square $R, F \cap R$ has only one component that connects $C$ to the boundary of $R$ (subsection 2.5.

We will show that the expected length of $F$ is at most a $\frac{4}{10} \epsilon$ fraction longer than OPT. By Markov's inequality, with probability at least one-half the length $(F) \leq\left(1+\frac{8}{10} \epsilon\right)$ OPT. We show that by moving the terminals back to 
their original positions (from their nearest integer coordinates) increases the length by at most $\frac{\epsilon}{40} \mathrm{OPT}$. Therefore, the output solution has length at most $(1+\epsilon)$ OPT with probability one half.

We now describe each of these steps in detail.

\subsection{Partition}

We first partition the set of terminal pairs, creating subproblems that can be solved independently of each other without loss of optimality. The purpose of this partition is to bound the size of the bounding box for each problem in terms of OPT. This bound is required for the next step, the result of which allows us to treat this geometric problem as a combinatorial problem. This discretization was also key to Arora's scheme, but the bound on the size of the bounding box for the problems he considers is trivially achieved. This is not the case for the Steiner forest problem. The size of the bounding box of all the terminals in an instance may be unrelated to the length of OPT.

Let $Q$ be the set of $m$ pairs $\left\{\left(t_{i}, t_{i}^{\prime}\right)\right\}_{i=1}^{m}$ of $n$ terminals. Consider the Euclidean graph whose vertices are the terminals and whose edges are the line segments connecting terminal pairs in $Q$ and let $C_{1}, C_{2}, \ldots$ be the components of this graph. Let $\operatorname{dist}(Q)=\max _{i} \operatorname{diam}\left(C_{i}\right)$; this is the maximum distance between any pair of terminals that must be connected.

Theorem 2.1. There exists a partition of $Q$ into independent instances $Q_{1}, Q_{2}, \ldots$ such that the optimal solution for $Q$ is the disjoint union of optimal solutions for each $Q_{i}$ and such that the diameter of $Q_{i}$ is at most $n_{i}^{2}$ dist $\left(Q_{i}\right)$ where $n_{i}$ is the number of terminals in $Q_{i}$. Further, this partition can be found in $O(n \log n)$ time.

We will show that the following algorithm, $\operatorname{PARTITION}(Q)$, produces such a partition. Let $T$ be the minimum spanning tree of the terminals in $Q$.

$\operatorname{Partition}(Q, T)$

Let $e$ be the longest edge of $T$.

If length $(e)>n \operatorname{dist}(Q)$,

remove $e$ from $T$ and let $T_{1}$ and $T_{2}$ be the resulting components.

For $i=1,2$, let $Q_{i}$ be the subset of terminal pairs connected by $T_{i}$.

$T:=\operatorname{PaRTITION}\left(Q_{1}, T_{1}\right) \cup \operatorname{PARTITION}\left(Q_{2}, T_{2}\right)$.

Return the partition defined by the components of $T$.

Proof of Theorem 2.1. First observe that by the cut property of minimum spanning trees, the distance between every terminal in $T_{1}$ and every terminal in $T_{2}$ is at least as long as the edge that is removed.

Since a feasible solution is given by the union of minimum spanning trees of the sets of the requirement partition, and each edge in these trees has length at most $\operatorname{dist}(Q)$, OPT $<n \operatorname{dist}(Q)$. OPT cannot afford to connect a terminal of $T_{1}$ to a terminal of $T_{2}$, because the distance between any terminal in $T_{1}$ and any terminal in $T_{2}$ is at least $n \operatorname{dist}(Q)$ which is greater than the lower bound. (By definition of dist, there cannot have been a requirement to connect a terminal of $T_{1}$ to a terminal of $T_{2}$.) Therefore, OPT must be the union of two solutions, one for the terminals contained by $T_{1}$ and one for the terminals contained by $T_{2}$. Inductively, the optimal solution for $Q$ is the union of optimal solutions for each set in PARTITION $(Q)$, giving the first part of the theorem.

The stopping condition of PARTITION guarantees that there is a spanning tree of the terminals in the current subset $Q_{i}$ of terminals whose edges each have length at most $n_{i} \operatorname{dist}\left(Q_{i}\right)$. Therefore, there is a path between each pair of terminals of length at most $n_{i}^{2} \operatorname{dist}\left(Q_{i}\right)$, giving the second part of the theorem.

Finally, we show that PARTITION can be implemented to run in $O(n \log n)$ time. The diameter of a set of points in the Euclidean plane can be computed by first finding a convex hull and this can be done in $O(n \log n)$ by, for example, Graham's algorithm [12]. Therefore, $\operatorname{dist}\left(C_{i}\right)$ can be computed in $O(n \log n)$ time. The terminal-pair sets $Q_{1}$ and $Q_{2}$ for the subproblems need not be computed explicitly as the required information is given by $T_{1}$ and $T_{2}$. By representing $T$ with a top-tree data structure, we can find $n_{i}$ and $d\left(Q_{i}\right)$ by way of a cut operation and a sum and maximum query, respectively, in $O(\log n)$ time [11]. Since there are $O(n)$ recursive calls, the total time for the top-tree operations is $O(n \log n)$.

Our PTAS finds an approximately optimal solution to each subproblem $Q_{i}$ (as defined by Theorem 2.1p and combines the solutions. For the remainder of our description of the algorithm, we focus on how the algorithm addresses 
one such subproblem $Q_{i}$. In order to avoid carrying over subscripts and arguments $Q_{i}$, $\operatorname{dist}\left(Q_{i}\right), n_{i}$ throughout the paper, from now on we will consider an instance given by $Q$, $\operatorname{dist}(Q)$, and $n$, and assume it has the property that the maximum distance between terminals, whether belonging to a requirement pair or not, is at most $n^{2} \operatorname{dist}(Q)$. OPT will refer to the length of the optimal solution for this subproblem.

\subsection{Discretize}

We would like to treat the terminals as discrete combinatorial objects. In order to do so, we assume that the coordinates of the terminals lie on an integer grid. We can do so by scaling the instance, but this may result in coordinates of unreasonable size. Instead, we scale by a smaller factor and round the positions of the terminals to their nearest half-integer coordinates.

\section{Scale}

We scale by a factor of

$$
\frac{40 \sqrt{2} n}{\epsilon \operatorname{dist}(Q)}
$$

Before scaling, OPT $\geq \operatorname{dist}(Q)$, the distance between the furthest pair of terminals that must be connected. After scaling we get the following lower bound:

$$
\mathrm{OPT} \geq \frac{40 \sqrt{2} n}{\epsilon}
$$

Before scaling, $\operatorname{diam}(Q) \leq n^{2} \operatorname{dist}(Q)$ by Theorem 2.1. After scaling we get the following upper bound on the diameter of the terminals:

$$
\operatorname{diam}(Q) \leq \frac{40 \sqrt{2} n^{3}}{\epsilon}
$$

Herein, OPT refers to distances in the scaled version.

\section{Round}

We round the position of each terminal to the nearest grid center. Additionally, we will search for a solution that only uses Steiner points that are grid centers. We call this constrained problem the rounded problem. The rounded problem may merge terminals (and thus, their requirements).

Lemma 2.2. A solution to the Steiner forest can be derived from an optimal solution to the rounded problem at additional cost at most $\frac{\epsilon}{40} O P T$.

Proof. Let $F$ be an optimal solution to the rounded problem. From this we build a solution to the original problem by connecting the original terminals to their rounded counterparts with line segments of length at most $1 / \sqrt{2}$, ie. half the length of the diagonal of a unit square. There are $n$ terminals, so the additional length is at most $n / \sqrt{2}$ which is at most $\frac{\epsilon}{40}$ OPT by Equation (1).

Let $F$ be an optimal solution to the rounded problem. We relate the number of intersections of $F$ with grid lines to length $(F)$. We will bound the cost of our restrictions to portals and cells with this relationship.

Lemma 2.3. There is a solution to the rounded problem of length $\left(1+\frac{1}{10} \epsilon\right)$ OPT that satisfies

$$
\sum_{\text {grid lines } \ell}|F \cap \ell| \leq 3 O P T \text {. }
$$

Proof. We build a solution $F$ to the rounded problem from OPT by replacing each line segment $e$ of OPT with a line segment $e^{\prime}$ that connects the half-integer coordinates that are nearest $e$ 's endpoints (breaking ties arbitrarily but 
consistently). Since the additional length needed for this transformation is at most twice (for each endpoint of $e$ ) the distance from a point to the nearest half-integer coordinate:

$$
\text { length }\left(e^{\prime}\right) \leq \text { length }(e)+\sqrt{2}
$$

Since OPT has at most $n$ leafs, OPT has fewer than $n$ Steiner points and so has fewer than $4 n$ edges. The additional length is therefore no greater than $4 \sqrt{2} n$. Combining with Equation (11), this is at most $\frac{1}{10} \epsilon \mathrm{OPT}$.

$F$ is composed of line segments whose endpoints are half-integer coordinates. Such a segment $S$ of length $s$ can cross at most $s$ horizontal grid lines and at most $s$ vertical grid lines. Therefore

$$
\sum_{\text {grid lines } \ell}|S \cap \ell| \leq 2 s
$$

and summing over all segments of $F$ gives

$$
\sum_{\text {grid lines } \ell}|F \cap \ell| \leq 2 \text { length }(F) \leq 2\left(1+\frac{1}{10} \epsilon\right) \text { OPT }<3 \text { OPT }
$$

where the last inequality follows from $\epsilon<1$.

From here on out, our goal is to find the solution that is guaranteed by Lemma 2.3 We will not be able to find this solution optimally, but will be able to find a solution within our error bound of $\epsilon$ OPT.

\subsection{Dissect}

The recursive dissection starts with an $L \times L$ box that encloses the terminals and where $L$ is at least twice as big as needed. This allows some choice in where to center the enclosing box. We make this choice randomly. This random choice is used in bounding the incurred cost, in expectation, of structural assumptions (Section 4.3) that help to reduce the size of the dynamic programming table.

Formally, let $L$ be the smallest power of 2 greater than $2 \cdot \operatorname{diameter}(Q)$. In combination with Equation 2 , we get the following upper bound on $L$ :

$$
L \leq \frac{160 \sqrt{2}}{\epsilon} n^{3}
$$

The $x$-coordinate (and likewise the $y$-coordinate) of the lower left corner of the enclosing box are chosen uniformly at random from the $L / 2$ integer coordinates that still result in an enclosing box. We will refer to this as the random shift.

As described in section 1.2, we perform a recursive dissection of this enclosing box. This can be done in $O(n \log n)$ time [7]. By our choice of $L$ and the random shift, this dissection only uses the grid lines. Since the recursive dissection stops with unit dissection squares, the quad-tree has depth $\log L$.

Consider a vertical grid line $\ell$. Since there are $L / 2$ values of the horizontal shift, and $2^{i-1}$ of these values will result in $\ell$ being a depth- $i$ dissection line, we get

$$
\operatorname{Prob}[\operatorname{depth}(\ell)=i]=2^{i} / L
$$

\subsection{Designate portals}

We designate a subset of the points on each dissection line as portals. We will restrict our search for feasible solutions that cross dissection lines at portals only. We use the portal constant $A$, where

$$
A \text { is the smallest power of two greater than } 30 \epsilon^{-1} \log L \text {. }
$$

Formally, for each vertical (resp. horizontal) dissection line $\ell$, we designate as portals of $\ell$ the points on $\ell$ with $y$-coordinates (resp. $x$-coordinates) which are integral multiples of

$$
\frac{L}{A 2^{\mathrm{depth}(\ell)}} \text {. }
$$

There are no portals on the sides of root dissection square, the bounding box. Since a square at depth $i$ has sidelength $L / 2^{i}$ and is bounded by 4 dissection lines at depth at most $i$, we get: 
Lemma 2.4. A dissection square has at most $4 A$ portals on its boundary.

Consider perpendicular dissection lines $\ell$ and $\ell^{\prime}$. A portal $p$ of $\ell$ may happen to be a point of $\ell^{\prime}$ (namely, the intersection point), but $p$ may not be a portal of $\ell^{\prime}$, that is, it may not be one of the points of $\ell^{\prime}$ that were designated according to the above definition.

The following lemma will be useful in Subsection 4.2 for technical reasons.

Lemma 2.5. For every dissection square $R$, the corners of $R$ are portals (except for the points that are corners of the bounding box).

Proof. Consider a square $R$ at depth $i$. Consider the two dissection lines that divide $R$ into $4 \ell$ and $\ell^{\prime}$. The depth of these lines is $i+1$. These lines restricted to $R$, namely $\ell_{R}=\ell \cap R$ and $\ell_{R}^{\prime}=\ell^{\prime} \cap R$, have length $L / 2^{i}$, a power of 2 . Portals are designated as integral multiples of $L /\left(2^{i+1} A\right)$, also a power of 2 and a $1 / 2 A$ fraction of the length of $\ell_{R}$ and $\ell_{R}^{\prime}$. It follows that the endpoints and intersection point of $\ell_{R}$ and $\ell_{R}^{\prime}$ are portals of these lines.

\subsection{Solve via dynamic programming}

In order to overcome the computational difficulty associated with maintaining feasibility (as illustrated in Figure 2), we divide each dissection square $R$ into a regular $B \times B$ grid of cells; $B$, which will be defined later, is $O(1 / \epsilon)$ and is a power of 2. Each cell of the grid is either coincident with a dissection square or is smaller than the leaf dissection squares. Consider parent and child dissection squares $R_{P}$ and $R_{C}$; a cell $C$ of $R_{p}$ encloses four cells of $R_{C}$.

The dynamic programming table for a dissection square $R$ will be indexed by two subpartitions (partition of a subset) of the portals and cells of $R$; one subpartition will encode the connectivity achieved by a solution within $R$ and the other will encode the connectivity required by the solution outside $R$ in order to achieve feasibility. The details are given in the next section.

\section{The Dynamic Program}

\subsection{The dynamic programming algorithm}

The dynamic program will only encode subsolutions that have low complexity and permit feasibility. We call such subsolutions conforming. We build a dynamic programming table for each dissection square. The table is indexed by valid configurations and the entry will be the best compatible conforming subsolution.

\section{Low complexity and feasible: conforming subsolutions}

Let $R$ be a dissection square or a cell, and let $F$ be a finite number of line segments of $R$. We say that $F$ conforms to $R$ if it satisfies the following properties:

- (boundary property) $|F \cap \partial R| \leq 4(D+1)$.

- (portal property) Every connected component of $F \cap \partial R$ contains a portal of $R$.

- (cell property) Each cell $C$ of $R$ intersects at most one connected component of $F$ that also intersects $\partial R$.

- (terminal property) If a terminal $t \in R$ is not connected to its mate by $F$ then it is connected to $\partial R$ by $F$.

The constant $D$ is defined in Equation 77 and is $O(1 / \epsilon)$. Note that the first three properties are those that bound the complexity of the allowed solutions and the last guarantees feasibility. We say that a solution $F$ recursively conforms to $R$ if it conforms to all descendents dissection squares of $R$ (including $R$ ). We say that a solution $F$ is conforming if it recursively conforms to the root dissection square with every terminal connected to its mate. It is a trivial corollary of the last property that a conforming solution is a feasible solution to the Steiner forest problem. We will restate and prove the following in Section 4, the remainder of this section will give a dynamic program that finds a conforming solution.

Theorem 3.1 (Structure Theorem). There is a conforming solution that has, in expectation over the random shift of the bounding box, length at most $\left(1+\frac{\epsilon}{4}\right) O P T$. 


\section{Indices of the dynamic programming table: valid configurations}

The dynamic programming table $\mathrm{DP}_{R}$ for a dissection square $R$ will be indexed by subpartitions of the portals and cells of $R$ that we call configurations. A configuration of $R$ is a pair $\left(\pi^{\text {in }}, \pi^{\text {out }}\right)$ with the following properties: $\pi^{\text {in }}$ is a subpartition of the cells and portals of $R$ such that each part contains at least one portal and at least one cell; $\pi^{\text {out }}$ is a coarsening of $\pi^{\text {in }}$. See Figure $3 \pi^{\text {in }}$ will characterize the behaviour of the solution inside $R$ while $\pi^{\text {out }}$ will encode what connections remain to be made in order to make the solution feasible. For a terminal $t \in R$, we use $C_{R}[t]$ to denote the cell of $R$ that contains $t$. We say a configuration is valid if it has the following properties:

- (compact) $\pi^{\text {in }}$ has at most $4(D+1)$ parts and contains at most $4(D+1)$ portals.

- (connecting) For every terminal $t$ in $R$ whose mate is not in $R, C_{R}[t]$ is in a part of $\pi^{\text {in }}$. For every pair of mated terminals $t, t^{\prime}$ in $R$, either $C_{R}[t]$ and $C_{R}\left[t^{\prime}\right]$ are in the same part of $\pi^{\text {out }}$ or neither $C_{R}[t]$ nor $C_{R}\left[t^{\prime}\right]$ are in $\pi^{\text {in }}$.

The connecting property will allow us to encode and guarantee feasible solutions. Since a dissection square has $4 A$ portals (Lemma 2.4) and $B^{2}$ cells, the first property bounds the number of configurations:

Lemma 3.2. There are at most $\left(4 A+B^{2}\right)^{O(D)}$ or $\left(\epsilon^{-2} \log n\right)^{O(1 / \epsilon)}$ compact configurations of a dissection square.

We will use the following notation to work with configurations: For a subpartition $\pi$ of $S$ and an element $x \in S$, we use $\pi[x]$ to denote the part of $\pi$ containing $x$ if there is one, and $\emptyset$ otherwise. For two subpartitions $\pi$ and $\pi^{\prime}$ of a set $S$, we use $\pi \vee \pi^{\prime}$ to denote the finest possible coarsening of the union of $\pi$ and $\pi^{\prime}$. If we eliminate the elements that are in partition $\pi^{\prime}$ but not in partition $\pi, \pi \vee \pi^{\prime}$ is a coarsening of $\pi^{\prime}$ and vice versa.

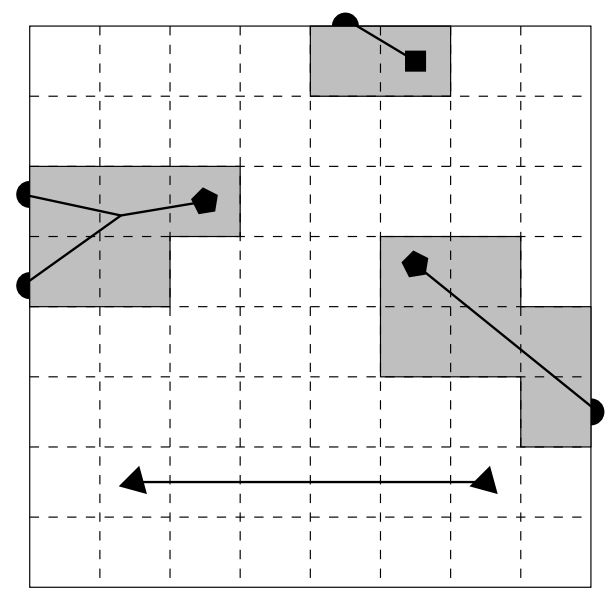

Figure 3: A dissection square and cells (grid), terminal pairs (triangles and pentagons) and unmated terminal (square), and subsolution (dark lines). The grey components give the parts of $\pi^{\text {in }}$ with portals (half-disks). To be a valid configuration, the two parts containing the pentagon terminals must be in the same part of $\pi^{\text {out }}$. The subsolution conforms to $R$ and is compatible with $\left(\pi^{\text {in }}, \pi^{\text {out }}\right)$.

\section{Entries of the dynamic programming table: compatible subsolutions}

The entries of the dynamic programming table for dissection square $R$ are compatible subsolutions, subsolutions that satisfy. Formally, a subsolution $F$ and configuration $\left(\pi^{\text {in }}, \pi^{\text {out }}\right)$ of $R$ are compatible if and only if $\pi^{\text {in }}$ has one part for every connected component of $F$ that intersects $\partial R$ and that part consists of the cells and portals of $R$ intersected by that connected component (Figure 3). Note that as a result, some valid configurations will not have a compatible subsolution: if a part of $\pi^{\text {in }}$ contains disconnected cells with terminals inside, then no set of line segments can connect these terminals and be contained by the cells of that part. The entries corresponding to such configurations will indicate this with $\infty$. 
Observation 3.3. If $F$ conforms to $R$ then $\left(\pi^{\text {in }}, \pi^{\text {out }}\right)$ is a valid configuration.

As is customary, our dynamic program finds the value of the solution; it is straightforward to augment the program so that the solution itself can be obtained. Our procedure for filling the dynamic programming tables, POPULATE, will satisfy the following theorem:

Theorem 3.4. POPULATE $(R)$ returns a table $D P_{R}$ such that, for each valid configuration $\left(\pi^{\text {in }}, \pi^{\text {out }}\right)$ of $R, D P_{R}\left[\pi^{\text {in }}, \pi^{\text {out }}\right]$ is the minimum length of subsolution that recursively conforms to $R$ and that is compatible with $\left(\pi^{\text {in }}, \pi^{\text {out }}\right)$.

We prove this theorem in Section 3.3 .

\section{Consistent configurations}

A key step of the dynamic program is to correctly match up the subsolutions of the child dissection squares $R_{1}, \ldots, R_{4}$ of $R_{0}$. Consider valid configurations $\left(\pi_{i}^{\text {in }}, \pi_{i}^{\text {out }}\right)$ for $i=0, \ldots, 4$ and let $\pi_{0}^{\vee}=\bigvee_{i=1}^{4} \pi_{i}^{\text {in }}$. We say that the configurations $\left(\pi_{i}^{\text {in }}, \pi_{i}^{\text {out }}\right)$ for $i=0, \ldots, 4$ are consistent if they satisfy the following connectivity requirements:

1. (internal) $\pi_{0}^{\text {in }}$ is given by $\pi_{0}^{\vee}$ with portals of $R_{i}$ that are not portals of $R_{0}$ removed, parts that do not contain portals of $R_{0}$ removed, and each cell of $R_{i}$ replaced by the corresponding (parent) cell of $R_{0}$. (If non-disjoint parts result from replacing cells by their parents, then the result is not a partition and cannot be $\pi_{0}^{\text {in }}$.)

2. (external) For two elements (cells and/or portals) $x, x^{\prime}$ of $R_{i}, \pi_{i}^{\text {out }}[x]=\pi_{i}^{\text {out }}\left[x^{\prime}\right]$ if and only if $\pi_{0}^{\vee}[x]=\pi_{0}^{\vee}\left[x^{\prime}\right]$ or there are portals $p, p^{\prime}$ such that $\pi_{0}^{\text {out }}[p]=\pi_{0}^{\text {out }}\left[p^{\prime}\right], \pi_{0}^{\vee}[x]=\pi_{0}^{\vee}[p]$, and $\pi_{0}^{\vee}\left[x^{\prime}\right]=\pi_{0}^{\vee}\left[p^{\prime}\right]$.

3. (terminal) For mated terminals $t \in R_{i}$ and $t^{\prime} \in R_{j}$ with $1 \leq i<j \leq 4$, either $\pi_{0}^{\vee}\left[C_{i}[t]\right]=\pi_{0}^{\vee}\left[C_{j}\left[t^{\prime}\right]\right]$ or $\pi_{0}^{\text {out }}\left[C_{0}[t]\right]=\pi_{0}^{\text {out }}\left[C_{0}\left[t^{\prime}\right]\right]$.

\section{Dynamic programming procedure}

We now give the procedure POPULATE that fills the dynamic programming tables. The top dissection square $R$ has a single entry, the entry corresponding to the configuration $(\emptyset, \emptyset)$. The desired solution is therefore given by $\mathrm{DP}_{R}[\emptyset, \emptyset]$ after filling the table $\mathrm{DP}_{R}$ with POPULATE $(R)$. The corresponding solution is conforming. The following procedure is used to populate the entries of $\mathrm{DP}_{R_{0}}$. The procedure is well defined when the tables are filled for dissection squares in bottom-up order.

$\operatorname{POPULATE}\left(R_{0}\right)$

If $R_{0}$ contains at most one terminal, then

For every valid configuration $\left(\pi^{\text {in }}, \pi^{\text {out }}\right)$ of $R_{0}$,

$\% R_{0}$ is a leaf dissection square

$\mathrm{DP}_{R_{0}}\left[\pi^{\mathrm{in}}, \pi^{\text {out }]}:=0\right.$

For every part $P$ of $\pi^{\text {in }}$,

if the cells of $P$ are connected and contain the portals (and terminal) of $P$,

$F_{P}:=$ minimum-length set of lines in the cells of $P$ that connects the portals in $P$ (and terminal, if in $P$ ),

$\mathrm{DP}_{R_{0}}\left[\pi^{\text {in }}, \pi^{\text {out }}\right]:=\mathrm{DP}_{R_{0}}\left[\pi^{\text {in }}, \pi^{\text {out }}\right]+$ length $\left(F_{P}\right)$;

otherwise, $\mathrm{DP}_{R_{0}}\left[\pi^{\text {in }}, \pi^{\text {out }}\right]:=\infty . \quad \%$ no subsolution conforms to $\pi^{\text {in }}, \pi^{\text {out }}$

Otherwise,

let $R_{1}, R_{2}, R_{4}, R_{4}$ denote the children of $R_{0}$.

$\% R_{0}$ is a non-leaf dissection square

For every valid configuration $\left(\pi_{0}^{\text {in }}, \pi_{0}^{\text {out }}\right)$ of $R_{0}$, initialize $\mathrm{DP}_{R_{0}}\left(\pi_{0}^{\text {in }}, \pi_{0}^{\text {out }}\right):=\infty$.

For every quintuple of indices $\left\{\left(\pi_{i}^{\text {in }}, \pi_{i}^{\text {out }}\right)\right\}_{i=0}^{4}$ to $\left\{\mathrm{DP}_{R_{i}}\right\}_{i=0}^{4}$,

$$
\begin{aligned}
& \text { if }\left\{\left(\pi_{i}^{\text {in }}, \pi_{i}^{\text {out }}\right)\right\}_{i=0}^{4} \text { are consistent, } \\
& \quad \mathrm{DP}_{R_{0}}\left[\pi_{0}^{\text {in }}, \pi_{0}^{\text {out }}\right]:=\min \left\{\mathrm{DP}_{R_{0}}\left[\pi_{0}^{\text {in }}, \pi_{0}^{\text {out }}\right], \sum_{i=1}^{4} \mathrm{DP}_{R_{i}}\left[\pi_{i}^{\text {in }}, \pi_{i}^{\text {out }}\right]\right\} .
\end{aligned}
$$




\subsection{Running time}

Since each part of $\pi^{\text {in }}$ contains $O(D)$ portals (since $\pi^{\text {in }}$ is compact), $F_{P}$ is a Steiner tree of $O(D)$ terminals (portals and possibly one terminal) among the cells of $\pi^{\text {in }}$. To avoid the cells that are not in $\pi^{\text {in }}$, we will require at $O\left(B^{2}\right)$ Steiner points. $F_{P}$ can be computed in time proportional to $B$ and $D$ (which are $O(1 / \epsilon)$ ) by enumeration. Since the number of compact configurations is polylogarithmic and since there are $O(n \log n)$ dissection squares, the running time of the dynamic program is therefore $O\left(n \log ^{\xi} n\right)$, where $\xi$ is a constant depending on $\epsilon$.

\subsection{Correctness (proof of Theorem 3.4)}

We prove Theorem 3.4 giving the correctness of our dynamic program, by bottom-up induction. In the following, we use the notation, definitions and conditions of POPULATE. The base cases of the induction correspond to dissection squares that contain at most one terminal. If any part $P$ of $\pi^{\text {in }}$ contains cells or portals that are disconnected, then there is no subsolution that is compatible with $\pi^{\text {in }}$ and $\mathrm{DP}_{R_{0}}\left[\pi_{0}^{\text {in }}, \pi_{0}^{\text {out }}\right]=\infty$ represents this. Otherwise the subsolution $F_{0}$ that is given by the union of $\left\{F_{P}:\right.$ part $P$ of $\left.\pi^{\text {in }}\right\}$ is compatible with $\pi^{\text {in }}$ by construction. Further $F_{0}$ satisfies the terminal property of conformance with $R_{0}$ by construction and the remaining properties since it is compatible with a valid conformation.

When $R_{0}$ contains more than one terminal, for a valid configuration $\left(\pi^{\text {in }}, \pi^{\text {out }}\right)$ of $R_{0}$, we must prove:

Soundness If $\mathrm{DP}_{R_{0}}\left[\pi_{0}^{\text {in }}, \pi_{0}^{\text {out }}\right]$ is finite then there is a subsolution $F_{0}$ that recursively conforms to $R_{0}$, is compatible with $\left(\pi_{0}^{\text {in }}, \pi_{0}^{\text {out }}\right)$ and whose length is $\mathrm{DP}_{R_{0}}\left[\pi_{0}^{\text {in }}, \pi_{0}^{\text {out }}\right]$.

Completeness Any minimal subsolution $F_{0}$ that recursively conforms to $R_{0}$ and is compatible with $\left(\pi_{0}^{\text {in }}, \pi_{0}^{\text {out }}\right)$ has length at least $\mathrm{DP}_{R_{0}}\left[\pi_{0}^{\text {in }}, \pi_{0}^{\text {out }}\right]$.

The proof of Theorem 3.4 follows directly from this. We will use the following lemma:

Lemma 3.5. Let $\left\{\left(\pi_{i}^{i n}, \pi_{i}^{\text {out }}\right)\right\}_{i=0}^{4}$ be consistent configurations for dissection square $R_{0}$ and child dissection squares $R_{1}, \ldots, R_{4}$. For $i=1, \ldots, 4$, let $F_{1}, \ldots, F_{4}$ be subsolutions that recursively conform to $R_{i}$ and are compatible with $\left(\pi_{i}^{\text {in }}, \pi_{i}^{\text {out }}\right)$. Then $\cup_{i=1}^{4} F_{i}$ recursively conforms to $R_{0}$ and is compatible with $\left(\pi_{0}^{\text {in }}, \pi_{0}^{\text {out }}\right)$.

Proof. Recall that $F_{0}$ is compatible with $\left(\pi_{0}^{\text {in }}, \pi_{0}^{\text {out }}\right)$ if $\pi_{0}^{\text {in }}$ has one part for every connected component of $F_{0}$ that intersects $\partial R_{0}$ and that part consists of the cells and portals intersected by that component. Consider a component $K$ of $F_{0}$ that intersects $\partial R_{0}$. There must be a child dissection square $R_{i}$ with a part of $\pi_{i}^{\text {in }}$ that consists of the cells and portals intersected by $K \cap R_{i}$. Consider all such parts $P_{j}, j=1, \ldots$. (Note that there may be more than one such part from a given child dissection square.) These parts belong to a part $P$ of $\pi_{0}^{\vee}$.

We argue that no other child configuration parts make up $P$. For a contradiction, suppose another part $P^{\prime}$ is in the make up of $P$. Since $\left(\pi_{0}^{\text {in }}, \pi_{0}^{\text {out }}\right)$ is consistent with the child configurations, $P^{\prime}$ cannot share a cell with any of $P_{j}$, $j=1, \ldots$ for otherwise $P$ would not survive the pruning given by the internal connectivity requirement of consistency. Therefore, $P^{\prime}$ must share a portal with some $P_{j}$; the corresponding parts $K^{\prime}$ and $K_{j}$ would therefore also share this portal, implying that $K \cap K^{\prime}$ is connected, a contradiction.

Again, by the internal connectivity requirement of consistency, $P$ is obtained from $P_{j}, j=1, \ldots$ by:

- Removing the portals that are not in $R_{0}$. The remaining portals are on $\partial R_{0}$, and $K$ connects them since $K_{j}$, $j=1, \ldots$ connect them by the inductive hypothesis.

- Each cell $C$ of $P_{j}$ is replaced by the parent cell, which entirely contains $C \cap K$.

Finally $P$ is not removed altogether since $K$ intersects $\partial R_{0}$ and this intersection must contain a portal of $R_{0}$. Therefore, there is a part of $\pi_{0}^{\text {in }}$ obtained from $P$ that contains all the cells and portals intersected by $K$.

\section{Proof of soundness}

If $\mathrm{DP}_{R_{0}}\left[\pi_{0}^{\text {in }}, \pi_{0}^{\text {out }}\right]$ is finite, then there must be entries $\operatorname{DP}_{R_{i}}\left[\pi_{0}^{\text {in }}, \pi_{0}^{\text {out }}\right]$ that are finite for $i=1, \ldots, 4$ and such that $\mathrm{DP}_{R_{0}}\left[\pi_{0}^{\text {in }}, \pi_{0}^{\text {out }}\right]=\sum_{i=1}^{4} \mathrm{DP}_{R_{i}}\left[\pi_{0}^{\text {in }}, \pi_{0}^{\text {out }}\right]$. Then, by the inductive hypothesis, for $i=1, \ldots, 4$, there is a subsolution 
$F_{i}$ that recursively conforms to $R_{i}$, has length $\mathrm{DP}_{R_{i}}\left[\pi_{i}^{\text {in }}, \pi_{i}^{\text {out }}\right]$, and is compatible with $\pi_{i}^{\text {in }}, \pi_{i}^{\text {out }}$. We simply define $F_{0}=\bigcup_{i=1}^{4} F_{i}$; by definition, $F_{0}$ has the desired length. By Lemma $3.5, F_{0}$ is compatible with $\left(\pi_{0}^{\text {in }}, \pi_{0}^{\text {out }}\right)$. We show that $F_{0}$ conforms to $R_{0}$ by illustrating the four properties of conformance.

$\mathbf{F}_{\mathbf{0}}$ satisfies the portal property $\quad$ Let $K$ be a component of $F_{0} \cap \partial R_{0}$. For some child $R_{i}$, the intersection of $K$ with $\partial R_{i} \cap \partial R_{0}$ is nonempty. Since $F_{i}$ satisfies the portal property, $K \cap \partial R_{i} \cap \partial R_{0}$ must also contain a portal; that portal is also a portal of $R_{0}$.

F $_{\mathbf{0}}$ satisfies the cell property Let $C$ be a cell of $R_{0}$ that is enclosed by child dissection square $R_{i}$. Suppose for a contradiction that two connected components $K_{1}$ and $K_{2}$ intersect both $C$ and $\partial R$. Then $K_{1} \cap R_{i}$ and $K_{2} \cap R_{i}$ must be connected components of $F_{i}$ that intersect cells $C_{1}$ and $C_{2}$, respectively, and $\partial R_{i}$, where $C_{1}$ and $C_{2}$ are child dissection squares of $C$. Since $F_{i}$ satisfies the cell property w.r.t. $R_{i}, C_{1} \neq C_{2}$ and these cells belong to parts $P_{1} \neq P_{2}$ of $\pi_{i}^{\text {in }}$. By the internal connectivity quirement of consistency, these cells would both get replaced by $C$, implying that $\pi_{0}^{\text {in }}$ has two parts containing the same cell, a contradiction.

F $_{0}$ satisfies the terminal property Consider a terminal $t$ in $R_{i}$ and $R_{0}$ such that $C_{R_{i}}[t]$ is in a part $P$ of $\pi_{i}^{\text {in }}$ (for otherwise, the terminal property follows from the inductive hypothesis). If $t$ 's mate is not in $R_{0}$, then, by the connecting property of valid configurations, $C_{R_{0}}[t]$ is in a part of $\pi_{0}^{\text {in }}$ and the terminal property follows from compatibility. So suppose $t$ 's mate, $t^{\prime}$ is in $R_{0}$ (and child $R_{j}$ ).

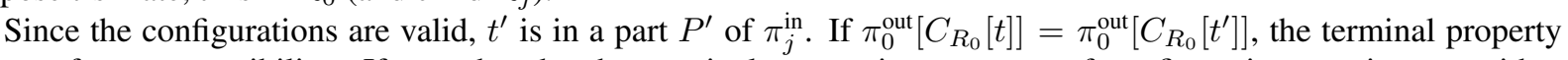
follows from compatibility. If not, then by the terminal connecting property of configuration consistency, either $\pi_{0}^{\vee}\left[C_{R_{i}}[t]\right]=\pi_{0}^{\vee}\left[C_{R_{j}}\left[t^{\prime}\right]\right]$. Since parts of child configurations cannot share cells, there must be a series of parts $P_{1}, \ldots, P_{k}$ where $P_{1}$ contains $C_{R_{i}}[t], P_{k}$ contains $C_{R_{j}}\left[t^{\prime}\right]$ and parts $P_{\ell}$ and $P_{\ell}+1$ contain a common portal $p_{\ell}$ for $\ell=1, \ldots, k-1$. Since $F_{1}, \ldots, F_{4}$ are compatible with $\pi_{1}^{\text {in }}, \ldots, \pi_{4}^{\text {in }}$, respectively, by the inductive hypothesis, there is a component $K_{\ell}$ in $\cup_{i=1}^{4} F_{i}$ that connects $t$ and $p_{1}$ (for $\ell=1$ ), $p_{\ell}$ and $p_{\ell+1}$ (for $\ell=2, \ldots, k-1$ ) and $p_{\ell}$ to $t^{\prime}$ (for $\ell=k) . \cup_{\ell=1}^{k} K_{\ell}$ is a component in $F_{0}$ that connects $t$ and $t^{\prime}$, giving the terminal property.

$\mathbf{F}_{0}$ satisfies the boundary property $\operatorname{Since}\left(\pi_{0}^{\text {in }}, \pi_{0}^{\text {out }}\right)$ is a valid configuration, $\pi_{0}^{\text {in }}$ has at most $4(D+1)$ parts. By compatibility, $F_{0}$ has at most $4(D+1)$ components intersecting $\partial R_{0}$. This proves the compactness property of conformance.

\section{Proof of completeness}

Let $\hat{F}_{0}$ be any minimal subsolution that recursively conforms to $R_{0}$ and is compatible with $\left(\pi_{0}^{\text {in }}, \pi_{0}^{\text {out }}\right)$. We show that $\hat{F}_{0}$ has length at least $\mathrm{DP}_{R_{0}}\left[\pi_{0}^{\text {in }}, \pi_{0}^{\text {out }}\right]$, proving completeness. For $i=1, \ldots, 4$, let $\hat{F}_{i}=\hat{F}_{0} \cap R_{i}$. Since $\hat{F}_{0}$ recursively conforms to $R_{0}, \hat{F}_{i}$ recursively conforms to $R_{i}$. For $i=1, \ldots, 4$, let $\left(\hat{\pi}_{i}^{\text {in }}, \hat{\pi}_{i}^{\text {out }}\right)$ be a configuration of $R_{i}$ that is compatible with $\hat{F}_{i}$. By Observation 3.3. $\left(\hat{\pi}_{i}^{\text {in }}, \hat{\pi}_{i}^{\text {out }}\right)$ is a valid configuration. By the inductive hypothesis, length $\left(\hat{F}_{i}\right) \geq \operatorname{DP}_{R_{i}}\left[\left(\hat{\pi}_{i}^{\text {in }}, \hat{\pi}_{i}^{\text {out }}\right)\right]$. It follows that length $\left(\hat{F}_{0}\right) \geq \sum_{i=1}^{4} \operatorname{DP}_{R_{i}}\left[\left(\hat{\pi}_{i}^{\text {in }}, \hat{\pi}_{i}^{\text {out }}\right)\right]$. If the child configurations $\left\{\left(\hat{\pi}_{i}^{\text {in }}, \hat{\pi}_{i}^{\text {out }}\right)\right\}_{i=1}^{4}$ are consistent with $\left(\pi_{0}^{\text {in }}, \pi_{0}^{\text {out }}\right), \sum_{i=1}^{4} \mathrm{DP}_{R_{i}}\left[\left(\hat{\pi}_{i}^{\text {in }}, \hat{\pi}_{i}^{\text {out }}\right)\right]$ will be an argument to the minimization in POPULATE and therefore length $\left(\hat{F}_{0}\right) \geq \mathrm{DP}_{R_{0}}\left[\pi_{0}^{\text {in }}, \pi_{0}^{\text {out }}\right]$. It is therefore sufficient to show that the child configurations $\left\{\left(\hat{\pi}_{i}^{\text {in }}, \hat{\pi}_{i}^{\text {out }}\right)\right\}_{i=1}^{4}$ are consistent with $\left(\pi_{0}^{\text {in }}, \pi_{0}^{\text {out }}\right)$. Equivalently, by Lemma 3.5 . $\hat{F}_{0}$ is compatible with the configuration $\left(\hat{\pi}_{0}^{\text {in }}, \hat{\pi}_{0}^{\text {out }}\right)$ that is consistent with $\left\{\left(\hat{\pi}_{i}^{\text {in }}, \hat{\pi}_{i}^{\text {out }}\right)\right\}_{i=1}^{4}$ according to the connectivity requirements of consistency.

This completes the proof of Theorem 3.4

\section{Proof of the Structure Theorem (Theorem 3.1)}

In this section we give a proof of the Structure Theorem (Theorem 3.1). We restate and reword the theorem here for convenience. It is easy to see that the statement here is equivalent to the statement given in Section 3, only the terminal property of conformance is missing, but that is encoded by feasibility. 
Theorem 3.1 (Structure Theorem). There is a feasible solution $F$ to the rounded Steiner forest problem having, in expectation over the random shift of the bounding box, length at most $\frac{2}{5} \epsilon$ OPT more than OPT such that each dissection square $R$ satisfies the following three properties:

Boundary Property For each side $S, F \cap S$ has at most D non-corner components, where

$$
D=60 \epsilon^{-1}
$$

Portal Property Each component of $F \cap \partial R$ contains a portal.

Cell Property For each cell $C$ of $R, F$ has at most one component that intersects both $\partial C$ and $\partial R$.

First, in a way similar to Arora, we illustrate the existence of a nearly-optimal solution that crosses the boundary of each dissection square a small number of times (Boundary Property) and does so at portals (Portal Property). To that end, starting with the solution $F_{0}$ as guaranteed by Lemma 2.3, we augment $F_{0}$ to create a solution $F_{1}$ that satisfies the Boundary Components Property, then augment $F_{1}$ to a solution $F_{2}$ that also satisfies the Portal Property. The Cell Property is then achieved by carefully adding to $F_{2}$ boundaries of cells that violate the Cell Property.

By Lemma 2.3, $F_{0}$ is longer than OPT by $\frac{\epsilon}{10} \mathrm{OPT}$. We show that we incur an additional $\frac{\epsilon}{10} \mathrm{OPT}$ in length in satisfying each of these three properties, for a total increase in length of $\frac{4}{10} \epsilon \mathrm{OPT}$, giving the Theorem.

\subsection{The Boundary Property}

We establish the Boundary Property constructively by starting with $F_{1}=F_{0}$ and adding closures of the intersection of $F_{1}$ with the sides of dissection squares. For a subset $X$ of a line, let closure $(X)$ denote the minimum connected subset of the line that spans $X$. For a side $S$ of a dissection square $R$, a connected component of a subset of $S$ is a non-corner component if it does not include a corner of $R$ The construction is a simple greedy bottom-up procedure:

\section{SATISFYBOUNDARY:}

For each $j$ decreasing from $\log L$ to 0 ,

For each dissection line $\ell$ such that $\operatorname{depth}(\ell) \leq j$,

for each $j$-square with a side $S \subseteq \ell$,

if $\mid$ non-corner components of $\left.F_{1} \cap S\right\} \mid>D$, add closure(non-corner components of $\left.F_{1} \cap S\right)$ to $F_{1}$.

\section{SATISFyBoundary establishes the Boundary Property}

Consider a dissection square $R$, a side $S$ of $R$, and the dissection line $\ell$ containing $S$. The iteration involving $\ell$ and $j=\operatorname{depth}(\ell)$ ensures that, at the end of that iteration, there are at most $D$ components of $F_{1} \cap S$ not including the endpoints of $S$, which are corners of $R$. We need to show that later iterations do not change this property.

Consider an iteration corresponding to $j^{\prime} \leq j$, a line $\ell^{\prime}$ with $j^{\prime} \geq \operatorname{depth}\left(\ell^{\prime}\right)$, and a side $S^{\prime} \subseteq \ell^{\prime}$ of a $j^{\prime}$-square $R^{\prime}$. By the nesting property and since $S^{\prime}$ cannot be enclosed by $S, S \cap \ell^{\prime}$ is either empty, a corner of $R$ or equal to $S$. In the first case, $S \cap F_{1}$ is not affected by adding a segment of $S^{\prime}$. In the second case, no new non-corner component of $F_{1} \cap S$ appears. In the third case, if adding a segment of $S^{\prime}$ would reduce $\left|S \cap F_{1}\right|$ to one. See Figure 4.

\section{The increase in length due to SATISFYBOUNDARY is small}

For iteration $j$ of the outer loop and iteration $\ell$ such that $j \geq \operatorname{depth}(\ell)$ of the second loop, let random variable $C_{\ell, j}$ denote the number of executions of the last step:

add closure (non-corner components of $\left.F_{1} \cap S\right)$ to $F_{1}$

Note that, conditioning on $\operatorname{depth}(\ell) \leq j, C_{\ell, j}$ is independent of $\operatorname{depth}(\ell)$ (however $C_{\ell, j}$ does depend on the random shift in the direction perpendicular to $\ell$ ). Initially the number of non-corner components of $F_{1} \cap \ell$ is at most the number of components, $\left|F_{0} \cap \ell\right|$. As argued above: for every $j \geq \operatorname{depth}(\ell)$, every $j$-square either is disjoint from $\ell$ or has a side on $\ell$, so dealing with a line $\ell^{\prime}$ parallel to $\ell$ does not increase the number of components on $\ell$; For every $j<\operatorname{depth}(\ell)$, dealing with a line $\ell^{\prime}$ perpendicular to $\ell$ can only introduce a corner component on $\ell$. So, the total 

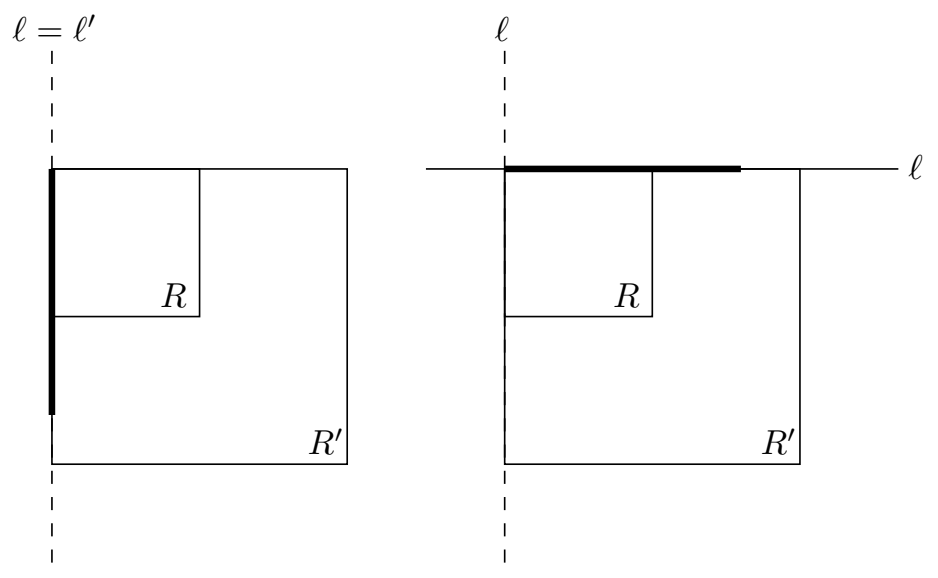

Figure 4: The second (right) and third (left) cases for showing that SATISFYBOUNDARY can only decrease the number of components along the side of another dissection square or adding a corner component when a segement (thick line) of a dissection square side $(R \cap \ell)$ is added to $F$ (not shown).

number of non-corner components on $\ell$ never increases. Since it decreases by $D$ at each of the $C_{\ell, j}$ closure operations, we have

$$
\sum_{j=\operatorname{depth}(\ell)}^{\log L} C_{\ell, j} \leq\left|F_{0} \cap \ell\right| / D .
$$

Since length $(S)=L / 2^{j}$, the total increase in length resulting from these executions is at most $C_{\ell, j}\left(L / 2^{j}\right)$. Therefore, the expected increase in length along $\ell$ is

$$
\begin{aligned}
E\left(\text { length }\left(F_{1} \cap \ell\right)-\operatorname{length}\left(F_{0} \cap \ell\right)\right) & \leq \sum_{i} \operatorname{Prob}[\operatorname{depth}(\ell)=i] \sum_{j \geq i} E\left[C_{\ell, j} \mid \operatorname{depth}(\ell)=i\right] \frac{L}{2^{j}} \\
& =\sum_{i} \frac{2^{i}}{L} \sum_{j \geq i} E\left[C_{\ell, j} \mid \operatorname{depth}(\ell) \leq j\right] \frac{L}{2^{j}} \\
& =\sum_{j} E\left[C_{\ell, j} \mid \operatorname{depth}(\ell) \leq j\right] \frac{1}{2^{j}} \sum_{i \leq j} 2^{i} \\
& \leq 2 E\left[\sum_{j \geq \operatorname{depth}(\ell)} C_{\ell, j} \mid \operatorname{depth}(\ell)\right] \\
& \leq 2\left|F_{0} \cap \ell\right| / D
\end{aligned}
$$

Summing over all dissection lines $\ell$, and using the bounds on $\sum_{\ell}\left|F_{0} \cap \ell\right|$ and $D$ as given by Equations (3) and (7), respectively, we infer that the length of $F_{1}$ is at most $\frac{\epsilon}{10}$ OPT more than the length of $F_{0}$.

\subsection{The Portal Property}

We establish the Portal Property constructively by starting with $F_{2}=F_{1}$ and extending $F_{2}$ along the boundaries of dissection squares to nearest portals. We say a component is portal-free if it does not contain a portal. The following construction establishes the Portal Property:

\section{SATISFYPORTAL:}

For each $j$ decreasing from $\log L$ to 0 ,

For each dissection line $\ell$ such that $\operatorname{depth}(\ell)=j$,

for each portal-free component $K$ of $F_{2} \cap \ell$, extend $K$ to the nearest non-corner portal on $\ell$. 


\section{SATISFY PORTAL preserves the Boundary Property}

Focus on dissection line $\ell$. Before the iteration corresponding to $\ell$, possible extensions along lines $\ell^{\prime}$ that are perpendicular to $\ell$ and of depth greater than of equal to $\operatorname{depth}(\ell)$ do not extend to $\ell$, because $\ell^{\prime} \cap \ell$ is a corner of $\ell^{\prime}$. After the iteration corresponding to $\ell$, for each possible extension along lines $\ell^{\prime}$ that are perpendicular to $\ell$ and of depth strictly less than $\operatorname{depth}(\ell), \ell^{\prime} \cap \ell$ is a corner of any dissection square $R$ with a side along $\ell$ containing $\ell \cap \ell^{\prime}$, so the Boundary Property for $\ell$ is not violated.

\section{The increase in length due to SATISFYPORTAL is small}

Consider a dissection line $\ell$. When dealing with line $\ell$, SATISFy PorTAL only merges components and, in doing so, does not increase the number of components of $F_{1} \cap \ell$. When dealing with a dissection line $\ell^{\prime}$ perpendicular to $\ell$, As SATISFYPORTAL might add the component $\ell \cap \ell^{\prime}$ to $F_{1} \cap \ell$. However, similar to the argument used above, in that case $\ell^{\prime} \cap \ell$ is a corner of any dissection square $R$ with a side along $\ell$ containing $\ell \cap \ell^{\prime}$. Since, by Lemma 2.5. corners are portals, no extension is made for this component. Therefore, each component of $F_{1} \cap \ell$ that does not already contain a portal is an extension of what was originally already a component of $F_{0} \cap \ell$ and so, at most $\left|F_{0} \cap \ell\right|$ extensions are made along $\ell$.

Each of these extensions adds a length of at most $L /\left(A 2^{\operatorname{depth}(\ell)}\right)$ (the inter-portal distance for line $\ell$ ). Therefore, the total length added along dissection line $\ell$ is bounded by $\left|F_{0} \cap \ell\right| L /\left(A 2^{\operatorname{depth}(\ell)}\right)$. Since $\operatorname{Prob}[\operatorname{depth}(\ell)=i]=2^{i} / L$, the expected increase in length due to dissection line $\ell$ is

$$
\sum_{i=1}^{\log L} \frac{2^{i}}{L}\left|F_{0} \cap \ell\right| \frac{L}{2^{i} A}=\frac{\left|F_{0} \cap \ell\right| \log L}{A}
$$

Summing over all dissection lines and using Equations (3) and (6), we infer that the length of $F_{2}$ is at most $\frac{\epsilon}{10} \mathrm{OPT}$ more than the length of $F_{1}$.

\subsection{The Cell Property}

We establish the Cell Property constructively by starting with $F_{3}=F_{2}$ and adding to $F_{3}$ boundaries of cells that violate the Cell Property. Let $C$ be a cell of a dissection square $R$. We say $C$ is happy with respect to the solution $F_{3}$ if there is at most one connected component of $F_{3}$ that touches both the interior of $C$ and $\partial R$. We cheer up an unhappy cell $C$ by adding to $F_{3}$ a subset $A$ of $\partial C$, as illustrated in Figure 5 .

$$
A\left(C, F_{3}\right)=\partial(C) \backslash\left\{\operatorname{sides} S \text { of } C: \operatorname{depth}(S)<\operatorname{depth}(C) \text { and } S \cap F_{3}=\emptyset\right\} .
$$

Recall that each cell $C$ of $R$ is either coincident with a dissection square that is a descendant of $R$ or is smaller than and enclosed by a leaf dissection squares that is a descendant of $R$. Definitions for the depth of a cell and its sides are inherited from the definitions of dissection-square depths and dissection-line depths.

Happiness of all cells, and therefore the Cell Property, is established by the following procedure:

\section{SATISFYCELLABSTRACT:}

While there is an unhappy cell $C$, add $A\left(C, F_{3}\right)$ to $F_{3}$.

Let $\mathcal{C}$ be the set of cells that we augment in the above procedure.

We claim that there is a function $h$ from the cells $\mathcal{C}$ to the components of $F_{0}$ (the original forest that we started with prior to the SATISFY procedures) that is injective and, such that, for a cell $C$ of dissection square $R, f(C)$ is a component of $F_{0}$ that intersects $\partial R$.

To define $h$, consider the following abstract directed forest $H$ whose vertices correspond to connected components of $F_{0}$ and whose edges correspond to augmentations made by SATISFYCELL (defined formally as follows). An augmentation for cell $C$ is triggered by the existence of at least two connected components $T, T^{\prime}$ of the current $F_{3}$ that both touch the interior of $C$ and the boundary of its associated dissection square $R$. Since the SATISFY procedures 


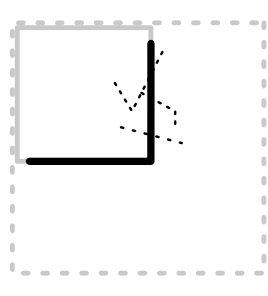

(a)

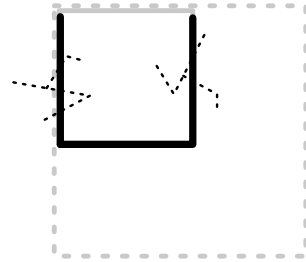

(b)

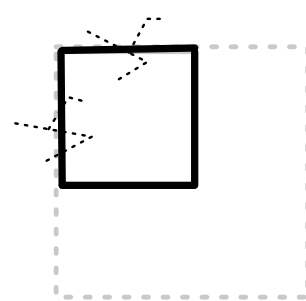

(c)

Figure 5: The three cases (up to symmetry) of augmenting $C$. The dotted lines are $F_{3}, C$ is the smaller square and $C$ 's parent is the larger square (to illustrate the relative depth of $C$ 's sides). In cases (a) and (b), the augmentation $A$ is not all of $\partial C$ so is open at the ends. In (a), $F_{3}$ intersects neither of the sides of $C$ that have depth less than that of $C$, so the augmentation $A$ consists only of the two sides having depth equal to that of $C$. In (b), one of the low-depth sides intersects $F_{3}$, so it belongs to $A$. In (c), both low-depth sides intersect $F_{3}$, so $A$ is all of $\partial C$.

augment the solution, $T$ and $T^{\prime}$ each contain (at least one) connected component $T_{0}$ and $T_{0}^{\prime}$ of $F_{0}-$ it is the vertices corresponding to $T_{0}$ and $T_{0}^{\prime}$ that are adjacent in $H$; we will show shortly that there exist such components that intersect $\partial R$. Arbitrarily root each tree of $H$ and direct each of its edges away from the root. For augmentation of cell $C$, we then define $h(C)$ as the component of $F_{0}$ that corresponds to the head of the edge of $H$ associated with the augmentation of $C$. Since each vertex of $H$ has indegree at most $1, h$ is injective.

We show, by way of contradiction, that there is a component of $F_{0}$ contained by $T$ that intersects $\partial R$. Consider all the components $\mathcal{T}$ of $F_{0}$ that are contained by $T$ and suppose none of these intersect $\partial R$. Let $\ell$ be a dissection line bounding $R$ that $T$ intersects. Since $\mathcal{T}$ does not intersect $\partial R, T$ must have been created from $\mathcal{T}$ by augmentations (by way of SATISFYBOUNDARY and SATISFYCELL) one of which added a subset $X$ of dissection line $\ell^{\prime}$ such that $X$ intersects $\ell$. Since $\mathcal{T}$ does not intersect $X$ and neither SATISFYBOUNDARY nor SATISFYCELL augment to the corner of a dissection line, $\ell$ and $\ell^{\prime}$ must be perpendicular. Further $X$ is a subset of a side $S^{\prime}$ of square $R^{\prime}$ and does not contain a corner of $R^{\prime}$. In summary, $R$ and $R^{\prime}$ are dissection squares bounded by perpendicular dissection lines $\ell$ and $\ell^{\prime}$ but for which $\ell \cap \ell^{\prime}$ is not a corner of $R^{\prime}$ or $R$, contradicting that dissection squares nest.

We are now ready to give an implementation of SATISFYCELLABSTRACT:

\section{SATISFYCELL:}

For each dissection line $\ell$,

for $j$ decreasing from $\log L$ to $\operatorname{depth}(\ell)$,

for each $j$-square $R$ with side $S \subseteq \ell$,

while there is an unhappy cell $C$ such that $h(C)$ intersects $\ell$ add $A\left(C, F_{3}\right)$ to $F_{3}$.

Since $h(C)$ intersects some side of some dissection square, this procedure makes each of the cells happy.

\section{The increase in length due to SATISFYCELL is small}

Let the random variable $C_{\ell, j}$ denote the number of augmentations corresponding to dissection line $\ell$ and index $j$. Thanks to the injective mapping $h$, we have:

$$
\sum_{j} C_{\ell, j} \leq\left|F_{0} \cap \ell\right| .
$$

Since a cell has boundary length shorter than its $j$-square by a factor of $B$, the total increase in length corresponding to these iterations is at most $C_{\ell, j}$ length $(j$-square $) / B$. Summing over $j$, the total length added by SATISFYCELL corresponding to dissection line $\ell$ is at most

$$
\sum_{j \geq \operatorname{depth}(\ell)} C_{\ell, j} \frac{4 L}{B 2^{j}} .
$$


Since the probability that grid line $\ell$ is a dissection line of depth $k$ is $2^{k} / L$, the expected increase in length added by SATISFYCELL corresponding to dissection line $\ell$ is at most

$$
\sum_{k} \frac{2^{k}}{L} \sum_{j \geq k} E\left[C_{\ell, j} \mid \operatorname{depth}(\ell)=k\right] \frac{4 L}{B 2^{j}} .
$$

As in Section 4.1. we observe that $C_{\ell, j}$ conditioned on $\operatorname{depth}(\ell) \leq j$ is independent of $\operatorname{depth}(\ell)$. By the same swapping of sums as before, this is then bounded by

$$
(8 / B) E\left[\sum_{j \geq \operatorname{depth}(\ell)} C(\ell, j) \mid \operatorname{depth}(\ell)\right] \leq \frac{8}{B}\left|F_{0} \cap \ell\right|
$$

Summing over all dissection lines, our bound on the expected additional length becomes

$$
\frac{8}{B} \sum_{\ell}\left|F_{0} \cap \ell\right|=\frac{24}{B}(1+\epsilon) \mathrm{OPT}
$$

For $B=240 / \epsilon$, this is at most $\frac{\epsilon}{10} \mathrm{OPT}$ by Equation (1).

\section{SATisfyCell maintains the Boundary and Portal Properties}

We show that SATISFyCell maintains the Boundary and Portal Properties by showing that for any forest $F$ satisfying the Boundary and Portal Properties, any single SATISFYCELL augmentation of $F$ also satisfies these properties.

Let $C$ be an unhappy cell and let $R$ be a dissection square satisfying the Boundary and Portal Properties. Let $A$ be the augmentation that is used to cheer up $C$. If $A \cap \partial R$ contains a corner of $R$, then the Boundary Property is satisfied because $A \cap \partial R$ would be a corner component and the Portal Property is satisfied because the corners of dissection squares are portals.

So, suppose that $A \cap \partial R$ is not empty but does not contain a corner of $R$. Refer to Figure 6 for relative positions of $R$ and $C$. Then $\partial C \cap \partial R$ cannot include an entire side of $R$, so it must be that $\operatorname{depth}(C)>\operatorname{depth}(R)$. Further, if $A \cap \partial R$ does not include a corner of $R$, then $A \cap \partial R$ must be a subset of a single dissection line, $\ell$.

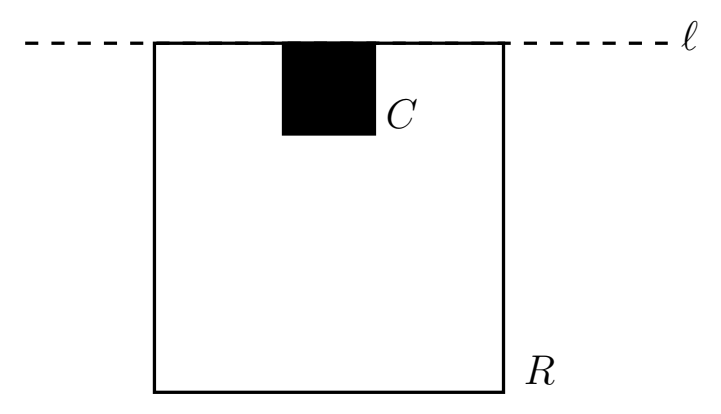

Figure 6: Relative positions of $R$ and $C$.

If $A \cap \ell \cap F$ is not empty, then $F \cap \ell$ is not empty. Since $F$ satisfies the Portal Property, $F \cap \ell$ also includes a portal. Since the addition of $A$ can only act to merge components, $|\ell \mathcal{R} \cap(\mathcal{F} \cup \mathcal{A})| \leq|\ell \mathcal{R} \cap \mathcal{A}|$ and so $F$ still satisfies the Boundary Property.

If $A \cap \partial R \cap F$ is empty, then, by Equation $(8), \operatorname{depth}(\ell) \geq \operatorname{depth}(C) . \operatorname{But} \operatorname{depth}(C)>\operatorname{depth}(R)$, so $\operatorname{depth}(\ell)>$ $\operatorname{depth}(R)$. This is impossible because $\ell$ is a line bounding $R$.

This completes the proof of Theorem 3.1 . 


\subsection{Proof of Theorem 1.1}

Recall Theorem 1.1, stating that there is a randomized $O(n \operatorname{polylog} n)$-time approximation scheme for the Steiner forest problem in the Euclidean plane. The proof of this Theorem is a corollary of Theorems 3.1, 3.4, 2.1 and Lemma 2.2 as follows. Theorem 3.4 guarantees that we can compute, using dynamic programming, a solution that satisfies Theorem 3.1. Section 3.2 argues that this DP takes $O(n$ polylog $n)$ time. Lemma 2.2 and Theorem 2.1 shows that we can convert the solution(s), of near-optimal cost, guaranteed by Theorem 3.1 to near-optimal solutions for the original problem, thus giving Theorem 1.1 .

\section{Conclusion}

We have given a randomized $O(n$ poly $\log n)$-time approximation scheme for the Steiner forest problem in the Euclidean plane. Previous to this result polynomial-time approximation schemes (PTASes) have been given for subsetTSP [14] and Steiner tree [9, 10] in planar graphs, using ideas inspired from their geometric counterparts. Since the conference version of this paper appeared, a PTAS has been given for Steiner forest in planar graphs by Bateni et al. [6]. Like our result here, Bateni et al. first partition the problem and then face the same issue of maintaining feasibility that we presented in Section 1.3. except in graphs of bounded treewidth. They overcome this by giving a PTAS for Steiner forest in graphs of bounded treewidth; they also show this problem in NP-complete, even in graphs of treewidth 3. Recently we have seen this technique generalized to prize collecting versions of the problem for both Euclidean and planar [5] instances.

\section{References}

[1] A. Agrawal, P. Klein, and R. Ravi. When trees collide: An approximation algorithm for the generalized Steiner problem on networks. SIAM Journal on Computing, 24(3):440-456, 1995.

[2] S. Arora. Polynomial-time approximation schemes for Euclidean TSP and other geometric problems. Journal of the ACM, 45(5):753-782, 1998.

[3] S. Arora. Approximation schemes for NP-hard geometric optimization problems: A survey. Mathematical Programming, 97:43-69, 2003.

[4] S. Arora, P. Raghavan, and S. Rao. Approximation schemes for Euclidean k-medians and related problems. In Proceedings of the 30th Annual ACM Symposium on Theory of Computing, pages 106-113, 1998.

[5] M. Bateni, C. Chekuri, A. Ene, M. Hajiaghayi, N. Korula, and D. Marx. Prize-collecting Steiner problems on planar graphs. In Proceedings of the 22nd Annual ACM-SIAM Symposium on Discrete Algorithms, pages 1028-1049, 2011.

[6] MohammadHossein Bateni, MohammadTaghi Hajiaghayi, and Dániel Marx. Approximation schemes for steiner forest on planar graphs and graphs of bounded treewidth. In Proceedings of the 42nd ACM symposium on Theory of computing, STOC '10, pages 211-220, New York, NY, USA, 2010. ACM.

[7] M. Bern, D. Eppstein, and S. Teng. Parallel construction of quadtree and quality triangulations. In Proceedings of the 3rd International Workshop on Algorithms and Data Structures, pages 188-199, 1993.

[8] M. Bern and P. Plassmann. The Steiner problem with edge lengths 1 and 2. Information Processing Letters, 32:171-176, 1989.

[9] G. Borradaile, C. Kenyon-Mathieu, and P. Klein. A polynomial-time approximation scheme for Steiner tree in planar graphs. In Proceedings of the 18th Annual ACM-SIAM Symposium on Discrete Algorithms, pages 1285-1294, 2007. 
[10] G. Borradaile, P. Klein, and C. Mathieu. An $O(n \log n)$ approximation scheme for Steiner tree in planar graphs. ACM Transactions on Algorithms, 5(3):1-31, 2009.

[11] A. Goldberg, M. Grigoriadis, and R. Tarjan. Use of dynamic trees in a network simplex algorithm for the maximum flow problem. Mathematical Programming, 50:277-290, June 1991.

[12] R. Graham. An efficient algorithm for determining the convex hull of a finite planar set. Information Processing Letters, 1:132-133, 1972.

[13] R. Karp. On the computational complexity of combinatorial problems. Networks, 5:45-68, 1975.

[14] P. Klein. A subset spanner for planar graphs, with application to subset TSP. In Proceedings of the 38th Annual ACM Symposium on Theory of Computing, pages 749-756, 2006.

[15] S. Kolliopoulos and S. Rao. A nearly linear-time approximation scheme for the Euclidean k-median problem. SIAM Journal on Computing, 37(3):757-782, 2007.

[16] J. Mitchell. Guillotine subdivisions approximate polygonal subdivisions: A simple polynomial-time approximation scheme for geometric TSP, k-MST, and related problems. SIAM Journal on Computing, 28(4):1298-1309, 1999.

[17] S. Rao and W. Smith. Approximating geometrical graphs via "spanners" and "banyans". In Proceedings of the 30th Annual ACM Symposium on Theory of Computing, pages 540-550, 1998.

[18] M. Thimm. On the approximability of the Steiner tree problem. In Proceedings of the 2th International Symposium on Mathematical Foundations of Computer Science, volume 2136 of Lecture Notes in Computer Science, pages $678-689,2001$.

[19] L. Trevisan. When Hamming meets Euclid: the approximability of geometric TSP and MST. SIAM Journal on Computing, 30(2):475-485, 2001.

[20] V. Vazirani. Approximation Algorithms, chapter Euclidean TSP, pages 84-89. Springer, 2001. 